\title{
Synthesis and Characterization a New 1,3-Diazepine Compounds from New Bis 4-Amino-3-Mercpto-1,2,4-Triazole Derivatives
}

\author{
Atyaf AQ. Younus ${ }^{1}$ and Nasreen R. Jber ${ }^{2 *}$ \\ ${ }^{1}$ Department of Chemistry, College of Education for Pure Science, University of Al-Anbar, \\ Anbar-Iraq. \\ ${ }^{2}$ Department of Chemistry, College of Science, Al-Nahrain University, Baghdad-Iraq. \\ Corresponding Author: Nasreen301174@yahoo.com
}

\begin{abstract}
Compounds with two triazole rings IVa,b were synthesized through the reaction of diacid hydrazide firstly with $\mathrm{CS}_{2} / \mathrm{KOH}$, and secondly with excess of hydrazine hydrate. The one-step reaction of isatin with benzylidene benzeneamine $\left(S_{1}-S_{10}\right)$ in ethanol gave 2-Ar-3Ar'[3,4b][1,3]benzodiazepine-4,5-diones $\left(\mathrm{D}_{1}-\mathrm{D}_{10}\right)$ in good yields. The products were characterized using FTIR, ${ }^{1}$ HNMR spectroscopy and CHN elemental analyses. [DOI: 10.22401/JNUS.20.2.01]
\end{abstract}

Keywords: 1,3-Diazepine, 1,2,4-Traiazole, Schiff s bases.

\section{Introduction}

Diazepines is a class of seven-membered ring heterocyclic compounds with two nitrogen atoms at 1,2-, 1,3- and 1,4- in the heptane ring instead of carbon atom and these compounds may be saturated and unsaturated with different substituents [1].

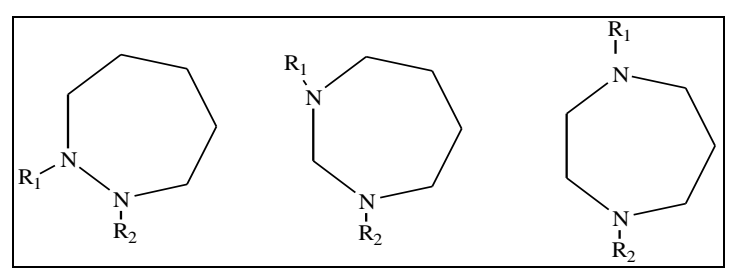

$[1,2]$ Diazepine [1,3] Diazepine [1,4]

Diazepine

The benzodiazepines are consisting of benzene ring fused with the diazepine ring to give the three analogous [1].

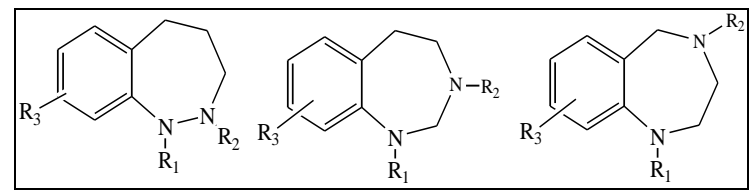

Benzo[1,2]diazepine Benzo[1,3] diazepine Benzo [1,4] diazepine

The biochemistry of diazepines and benzodiazepines has been thoroughly and extensively explored owing to their association with wide spectrum of pharmacological activities, such as, sedatives, anxiolytics, hypnotics, anticonvulsants, antipsychotics, and muscle relaxants [2].
Some 1,3-diazepin-2-ones and other cyclic ureas have received considerable attention recently as potential anti-AIDS drugs [3]. In Fig.(1) for example chlorodiazepoxide (1) known as the first benzodiazepine, was coincidentally available in markets in 1960. It is very important derivative in terms of hypnotic, anxiolytic and muscle relaxant [4]. Diazepam (valium) (2) was also discovered to show better activity in psychotherapy. Then, another derivative of nitrazepam (3) was used against anypnia [5]. Similarly, clonazepam (4) has anxiolytic, anticonvulsant, muscle relaxant and hypnotic properties [6]. Additionally, it was recognized by the Food and Drug Administration (FDA) for treatment of epilepsy $[7,8]$
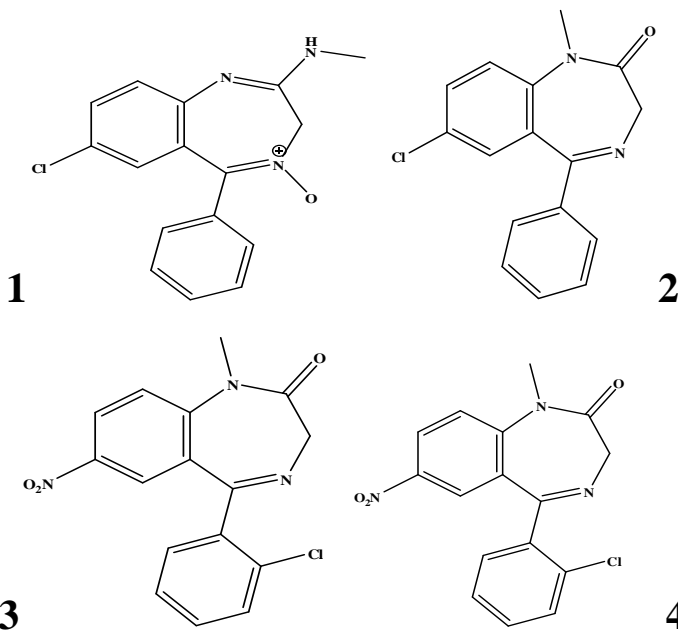

Fig. (1) 


\section{Experimental}

\section{1- Materials and apparatus}

All the chemicals used were supplied form BDH, Merck AND Fluka.

FTIR spectra in the range $(4000-400) \mathrm{cm}^{-1}$ were recorded using potassium bromide disc on FTIR instrument Model 8300 Shimadzu Spectrophotometer, Japan. ${ }^{1}$ HNuclear Magnetic Resonance (NMR) spectra were recorded on Brüker ACF 300 spectrometer at $300 \mathrm{MHz}$, using deutrated chloroform or DMSO as a solvent with TMS was used as an internal standard in University of Ahal Al-Bait, Jordon. Microanalyses were carried out on Euro EA Elemental analyzer A- 3000. Uncorrected melting points were recorded on hot stage Gallenkamp melting point apparatus (U.K.).

\section{2- Procedures}

\section{Succinic and Terephthalic dihydrazide}

Dimethyl ester of Succinic or Terephthalic $(0.025 \mathrm{~mol})$ in $25 \mathrm{ml}$ of ethanol was taken in round bottom flask. To that hydrazine hydrate (6ml) was added and refluxed for (4 hours). After cooling the crystals was precipitated out and recrystallized from ethanol to give compounds (II)a yield $89 \%$, m.p. $=170-172^{\circ} \mathrm{C}$, and (II)b yield $88 \%$, m.p. $=132-135^{\circ} \mathrm{C}$.

\section{Succinic and Terephthalic dihydrazide [Bis (potassium dithiocarbazinate)]}

To a solution of potassium hydroxide $(0.04 \mathrm{~mol})$ in ethanol absolute $(150 \mathrm{ml})$, dihydrazides (II) $)_{\mathrm{a}, \mathrm{b}}(0.02 \mathrm{~mol})$ and carbon disulfide $(3 \mathrm{ml})$ were added dropwise and the mixture was stirred in ice bath $\left(0-6^{\circ} \mathrm{C}\right)[6]$. The yellow solid was precipitated out and the potassium salt obtained in quantitative yield was directly used without purification.

\section{Bis [(3,3 'thio-4,4'amino-1,2,4-triazole $)-5$ - yl] ethane [9]}

A mixture of potassium salt and Hydrazine hydrate $(2 \mathrm{ml})$ and water $(80 \mathrm{ml})$ was refluxed for $4 \mathrm{hrs}$., the color of the reaction mixture changed to green, hydrogen sulfide was evolved and a homogenous solution resulted. A white solid was precipitated by dilution with cold water $(100 \mathrm{ml})$ and acidification with concentrated hydrochloric acid. The product was filtered, washed with cold water and recrystallized from ethanol, compound (IV) yield $72 \%$, m.p. $=220-222^{\circ} \mathrm{C}$.

Bis $\left[\left(3,3\right.\right.$-thio-4,4 ${ }^{\prime}$-amino-1,2,4-triazole $)-5$ yl] phenyl

A suspension of potassium salt of terephthalic dihydrazide $(2 \mathrm{~g}, 0.005 \mathrm{~mol})$, hydrazine hydrate $(2 \mathrm{ml}, 0.04 \mathrm{~mol})$ and water $(100 \mathrm{ml})$ was refluxed for $(3 \mathrm{hrs})$. The reaction mixture color was changed to light green, hydrogen sulfide was evolved and a homogenous solution resulted A solid was precipitated by dilution with cold water $(100 \mathrm{ml})$ and acidification with concentrated hydrochloric acid. The product was filtered, washed with cold water dried and recrystallized from ethanol, compound (IV) yield $82 \%$, m.p. $=198-200^{\circ} \mathrm{C}$.

\section{General procedure for preparation of Schiff bases [10]}

A mixture of triazole derivative (IVa-b) (0.001 mol) and the corresponding aldehydes $(0.002 \mathrm{~mol})$ in ethanol $(25 \mathrm{ml})$ was treated with glacial acetic acid $(0.5 \mathrm{ml})$ and refluxed for $(3 \mathrm{hr})$. The reaction mixture on cooling was filtered and recrystallized from ethanol. 
Table (1)

Physical properties of compounds $\left(S_{1-} S_{10}\right),\left[G=C_{2} H_{2}-C_{2}\right.$ or phenyl, $\left.\mathrm{Ar}=\mathrm{C}_{6} \mathrm{H}_{4}-\mathrm{CH}_{3}, \mathrm{C}_{6} \mathrm{H}_{4}-\mathrm{OCH} \mathrm{CH}_{3}, \mathrm{C}_{6} \mathrm{H}_{4}-\mathrm{Cl}, \mathrm{C}_{6} \mathrm{H}_{4}-\mathrm{Br}, \mathrm{C}_{6} \mathrm{H}_{4}-\mathrm{NO}_{2}, \mathrm{C}_{8} \mathrm{H}_{7}\right]$.

\begin{tabular}{|c|c|c|c|}
\hline Comp No. & m.p. ${ }^{\circ} \mathbf{C}$ & $\vee \mathrm{CH}=\mathrm{N}$ & Other \\
\hline $\mathrm{S}_{1}$ & $288-290$ & 3100 & $\begin{array}{c}1554 \\
1345\left(\mathrm{NO}_{2}\right) \\
\end{array}$ \\
\hline$\overline{\mathrm{S}_{2}}$ & $212-215$ & 3110 & - \\
\hline$\overline{\mathrm{S}_{3}}$ & $150-153$ & 3114 & $2987 \& 2877$ (C-H Aliphatic \\
\hline$\overline{\mathrm{S}_{4}}$ & $165-170$ & 3100 & $1100(\mathrm{C}-\mathrm{O})$ \\
\hline$\overline{\mathrm{S}_{5}}$ & $211-215$ & 3110 & - \\
\hline $\mathrm{S}_{6}$ & $232-235$ & 3102 & $\begin{array}{c}15564 \\
1353\left(\mathrm{NO}_{2}\right) \\
\end{array}$ \\
\hline $\mathrm{S}_{7}$ & $217-220$ & 3098 & $2997 \& 2854$ (C-H Aliphatic \\
\hline $\mathrm{S}_{8}$ & $140-143$ & 3097 & $2965 \& 2878$ (C-H Aliphatic \\
\hline$\overline{\mathrm{S}_{9}}$ & $145-147$ & 3112 & $2987 \& 2877$ (C-H Aliphatic \\
\hline $\mathrm{S}_{10}$ & $142-144$ & 3110 & 2981\& 2876 (C-H Aliphatic \\
\hline
\end{tabular}

General procedure for synthesis of substituted $[1,2,4]$ triazolo $[3,4-b][1,3]$ diazepine

A mixture of corresponding Schiff bases $\left(\mathrm{S}_{1}-\mathrm{S}_{10}\right)(0.001 \mathrm{~mol})$ and $(0.002 \mathrm{~mol})$ of isatine

Table (2)

Physical properties of compounds $\left(\mathrm{DI}-\mathrm{D}_{11}\right)\left[\mathrm{G}=\mathrm{CH}_{2}-\mathrm{CH}_{2}\right.$ or phenyl, $\left.\mathrm{Ar}=\mathrm{C}_{6} \mathrm{H}_{4}-\mathrm{CH}_{3}, \mathrm{C}_{6} \mathrm{H}_{4}-\mathrm{OCH}_{3}, \mathrm{C}_{6} \mathrm{H}_{4}-\mathrm{Cl}, \mathrm{C}_{6} \mathrm{H}_{4}-\mathrm{Br}, \mathrm{C}_{6} \mathrm{H}_{4}-\mathrm{NO}_{2}, \mathrm{C}_{8} \mathrm{H}_{7}\right]$.

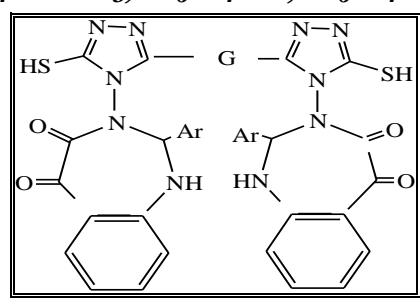

\begin{tabular}{|c|c|c|c|c|c|}
\hline Comp. No. & mp. & $\boldsymbol{v}(\mathbf{N}-\mathbf{H})$ & $\begin{array}{c}v(\mathrm{C}=\mathrm{O}) \\
\text { ketone }\end{array}$ & $\begin{array}{l}v(\mathrm{C}=\mathrm{O}) \\
\text { lactome }\end{array}$ & Others \\
\hline$D_{1}$ & $280-283$ & 3280 & 1724 & 1693 & - \\
\hline $\mathrm{D}_{2}$ & +280 & 3282 & 1726 & 1693 & - \\
\hline$D_{3}$ & $182-185$ & 3398 & 1714 & 1672 & $v(\mathrm{C} 28 \mathrm{Cl})$ \\
\hline $\mathrm{D}_{4}$ & $123-125$ & 3219 & 1721 & 1686 & $\begin{array}{c}3078 \vee(\mathrm{C}-\mathrm{H}) \\
\text { Arom. }\end{array}$ \\
\hline$D_{5}$ & $140-142$ & 3268 & 1732 & 1685 & $\begin{array}{c}1089 \\
v(\mathrm{C}-\mathrm{O}) \\
\end{array}$ \\
\hline $\mathrm{D}_{6}$ & & 3307 & 1729 & 1643 & $\begin{array}{c}1083 \\
v(\mathrm{C}-\mathrm{O}) \\
\end{array}$ \\
\hline $\mathrm{D}_{7}$ & +280 & 3310 & 1722 & 1658 & $\begin{array}{c}3082 \vee(\mathrm{C}-\mathrm{H}) \\
\text { Arom. }\end{array}$ \\
\hline $\mathrm{D}_{8}$ & 261-264 & 3263 & 1726 & 1660 & $\begin{array}{c}1552 \& 1325 v \\
\left(\mathrm{NO}_{2}\right)\end{array}$ \\
\hline $\mathrm{D}_{9}$ & $275-277$ & 3238 & 1739 & 1700 & $\begin{array}{c}1558 \& 1339 \\
V\left(\mathrm{NO}_{2}\right) \\
\end{array}$ \\
\hline $\mathrm{D}_{10}$ & $248-250$ & 3196 & 1719 & 1649 & $\begin{array}{c}931 \\
v(\mathrm{C}-\mathrm{Br}) \\
\end{array}$ \\
\hline $\mathrm{D}_{11}$ & $265-268$ & 3129 & 1721 & 1659 & $\begin{array}{c}985 \\
v(\mathrm{C}-\mathrm{Br}) \\
\end{array}$ \\
\hline
\end{tabular}




\section{Results and Discussion}

Synthesis of substituted [1,2,4] triazolo [3,4-

b] [1,3] diazepine $\left(D_{1}-D_{11}\right)$ :

The title compounds were synthesized according to the following scheme:

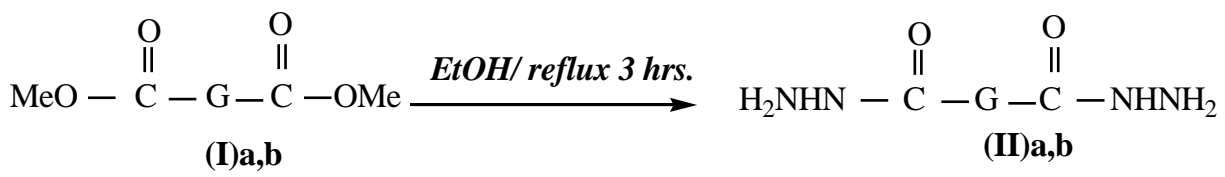

\section{$\mathrm{KOH} / \mathrm{CS}_{2}$}

EhOH

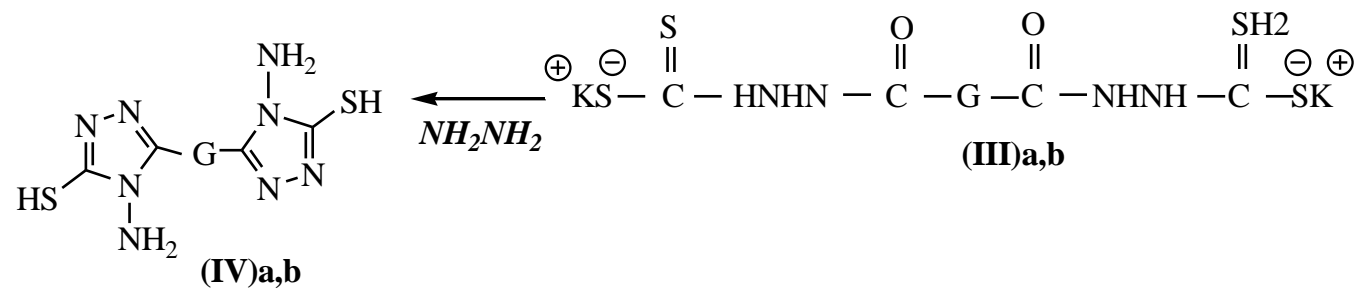

bis-(3,3'thio-4,4'amino-1,2,4-triazole)

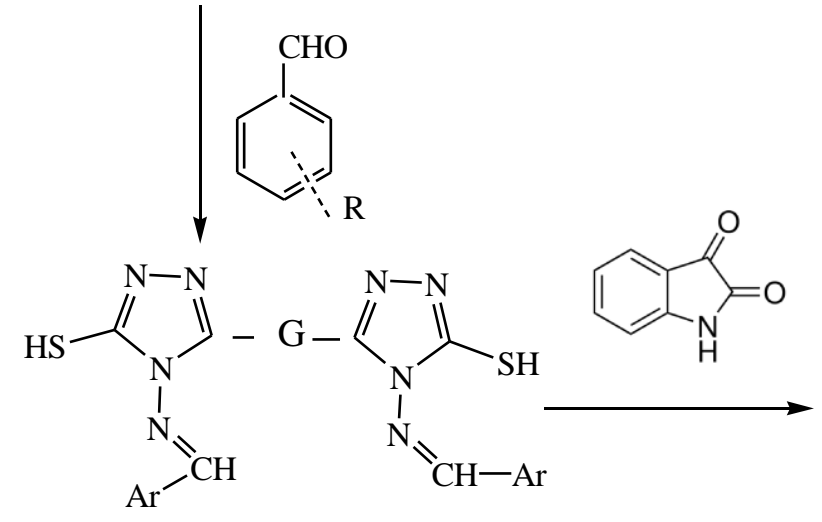

$\left(\mathbf{S}_{1}-\mathbf{S}_{10}\right)$

Bis[(3,3 -thio-4,4-benzelidineamino-1,2,4-triazole)-5-yl]

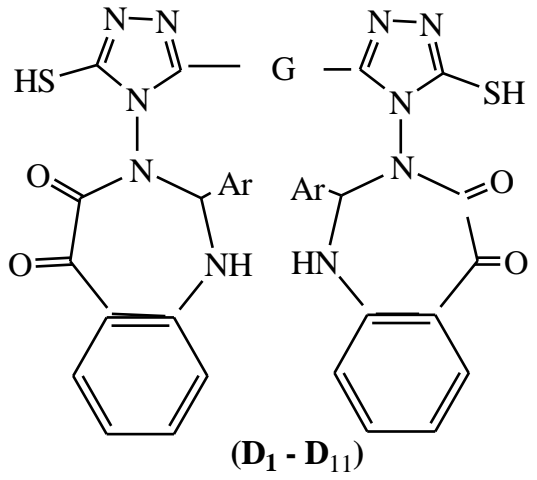

$[1,2,4]$ triazolo $[3,4-b][1,3]$ diazepine

$$
\mathrm{G}=-\mathrm{Ph} \quad \text { or } \quad-\mathrm{CH}_{2} \mathrm{CH}_{2}-
$$

Ar- : - $\mathrm{C}_{6} \mathrm{H}_{4}-\mathrm{CH}_{3}, \mathrm{C}_{6} \mathrm{H}_{4}-\mathrm{OCH}_{3}, \mathrm{C}_{6} \mathrm{H}_{4}-\mathrm{Cl}, \mathrm{C}_{6} \mathrm{H}_{4}-\mathrm{Br}, \mathrm{C}_{6} \mathrm{H}_{4}-\mathrm{NO}_{2}, \mathrm{C}_{8} \mathrm{H}_{7}$

Scheme (1): The synthetic pathway for [3,4-b][1,3] diazepine compounds $\left(D_{1}\right.$. $\left.D_{11}\right)$.

Compounds $(\mathrm{I})_{\mathrm{a}, \mathrm{b}}$ were prepared from direct esterfication of succinic acid and terphthalic acid with methanol in acidic media ${ }^{(11)}$. Compounds (II) $)_{a, b}$ were prepared from the reaction of esters $(\mathrm{I})_{\mathrm{a}, \mathrm{b}}$ with hydrazine hydrate [12].

The reaction of hydrazide compounds (II) $)_{a, b}$ with carbon disulfide in basic media leads to the formation of dithio carbazinate salts (III) $)_{\mathrm{a}, \mathrm{b}}$ which undergo cyclization in excess of hydrazine hydrate to give 3,3`-thio-4,4 amino-1,2,4-triazole (IV) $)_{\mathrm{a}, \mathrm{b}}$.

The structures of compounds (IV) $)_{\mathrm{a}}$ and $(\mathrm{IV})_{\mathrm{b}}$ were characterized using FTIR, ${ }^{1} \mathrm{H}-\mathrm{NMR}$ spectroscopic technique. The purity of final compounds were confirmed by using an elemental analysis. The elemental analysis of compounds (IV) $)_{\mathrm{a}, \mathrm{b}}$ are listed in Table (3). 
Table (3)

Elemental analysis (CHNS-O) for compounds $(I V)_{a, b}$.

\begin{tabular}{|c|c|c|c|c|c|c|c|c|c|}
\hline \multirow{2}{*}{ Comp. No. } & \multirow{2}{*}{ Formula } & \multicolumn{2}{|c|}{$\% \mathrm{C}$} & \multicolumn{2}{|c|}{$\% \mathrm{H}$} & \multicolumn{2}{|c|}{$\% \mathrm{~N}$} & \multicolumn{2}{|c|}{$\% \mathrm{~S}$} \\
\hline & & Calc. & Found & Calc. & Found & Calc. & Found & Calc. & Found \\
\hline$(\mathbf{I V})_{\mathrm{a}}$ & $\mathrm{C}_{10} \mathrm{H}_{10} \mathrm{~N}_{8} \mathrm{~S}_{2}$ & 39.21 & 39.98 & 3.26 & 4.17 & 36.60 & 36.84 & 20.91 & 18.93 \\
\hline$(I V)_{b}$ & $\mathrm{C}_{6} \mathrm{H}_{10} \mathrm{~N}_{8} \mathrm{~S}_{2}$ & 27.90 & 29.05 & 3.87 & 4.12 & 43.41 & 42.09 & 24.80 & 23.07 \\
\hline
\end{tabular}

The FTIR spectrum of compound (IV) $\left(\mathrm{KBr}\right.$ disc $\left.\mathrm{cm}^{-1}\right)$, show the appearance of bands at 3450, 3272, 3165, 3055, 1625, 1598 and 825 which could be assigned to $v \mathrm{~N}-\mathrm{H}$ (asymm. and symm.), $v \mathrm{C}-\mathrm{H}$ (aromatic), $v \mathrm{C}=\mathrm{N}, v \mathrm{C}=\mathrm{C}$ and out of plane bending of para- disustituted benzene ring.

Compounds $\left(\mathrm{S}_{1}-\mathrm{S}_{10}\right)$ were synthesized by the reaction of terephthalic or succinic dihydrazide [Bis(potassium dithiocarbazinate)] III $_{\mathrm{a}, \mathrm{b}}$ with hydrazine hydrate to achieve $\mathrm{Bis}$ [(3,3`-thio-4,4'-amino-1,2,4-triazole)-5-yl] ethane or phenyl IVa,b followed by condensation reaction with different aromatic aldehyde to achieve $\operatorname{Bis}[(3,3$ '-thio-4,4`benzelidineamino-1,2,4-triazole)-5-yl]ethane or phenyl $\left(\mathrm{S}_{1}-\mathrm{S}_{10}\right)$.

The structures of all products were identified using FT-IR and ${ }^{1} \mathrm{H}-\mathrm{NMR}$ for some of theme. All resultant spectral data were in correspondence with expected values. The purity of compounds were confirmed by using an elemental analysis. The elemental analysis of compounds $\left(\mathrm{S}_{1}-\mathrm{S}_{10}\right)$ are listed in Table (4). The observed values are in well agreement with theoretical values indicating structure of respective compounds.

Table (4)

Elemental analysis (CHNS-O) for compounds $\left(S_{1}-S_{10}\right)$.

\begin{tabular}{|c||l||c|c||c|c||c|c|c|c||}
\hline \multirow{2}{*}{$\begin{array}{c}\text { Comp. } \\
\text { No. }\end{array}$} & \multirow{2}{*}{ Formula } & \multicolumn{2}{c|}{ \%C } & \multicolumn{2}{c|}{ \% H } & \multicolumn{2}{c|}{ \% N } & \multicolumn{2}{c|}{ \% S } \\
\cline { 3 - 10 } & & Calc. & Found & Calc. & Found & Calc. & Found & Calc. & Found \\
\hline \hline $\mathrm{S}_{1}$ & $\mathrm{C}_{24} \mathrm{H}_{16} \mathrm{~N}_{10} \mathrm{O}_{4} \mathrm{~S}_{2}$ & 50.34 & 49.88 & 5.55 & 4.99 & 24.47 & 25.02 & 11.18 & 10.96 \\
\hline \hline $\mathrm{S}_{2}$ & $\mathrm{C}_{24} \mathrm{H}_{16} \mathrm{~N}_{8} \mathrm{~S}_{2} \mathrm{Br}_{2}$ & 45.01 & 45.34 & 2.50 & 2.61 & 17.50 & 18.01 & 10.00 & 9.78 \\
\hline \hline $\mathrm{S}_{3}$ & $\mathrm{C}_{26} \mathrm{H}_{22} \mathrm{~N}_{8} \mathrm{~S}_{2}$ & 61.17 & 61.67 & 4.31 & 4.66 & 21.96 & 22.21 & 12.54 & 12.87 \\
\hline \hline $\mathrm{S}_{4}$ & $\mathrm{C}_{26} \mathrm{H}_{22} \mathrm{~N}_{8} \mathrm{O}_{2} \mathrm{~S}_{2}$ & 57.56 & 56.89 & 4.05 & 4.21 & 20.66 & 19.88 & 11.80 & 11.75 \\
\hline $\mathrm{S}_{5}$ & $\mathrm{C}_{32} \mathrm{H}_{22} \mathrm{~N}_{8} \mathrm{~S}_{2}$ & 65.97 & 65.11 & 3.78 & 4.34 & 19.24 & 20.23 & 10.99 & 11.09 \\
\hline $\mathrm{S}_{6}$ & $\mathrm{C}_{20} \mathrm{H}_{16} \mathrm{~N}_{10} \mathrm{O}_{4} \mathrm{~S}_{2}$ & 45.80 & 46.08 & 3.05 & 2.99 & 26.71 & 26.05 & 12.21 & 12.45 \\
\hline \hline $\mathrm{S}_{7}$ & $\mathrm{C}_{20} \mathrm{H}_{16} \mathrm{~N}_{8} \mathrm{~S}_{2} \mathrm{Br}_{2}$ & 40.55 & 39.43 & 2.70 & 2.45 & 18.92 & 19.12 & 10.81 & 11.12 \\
\hline \hline $\mathrm{S}_{8}$ & $\mathrm{C}_{22} \mathrm{H}_{22} \mathrm{~N}_{8} \mathrm{~S}_{2}$ & 57.14 & 56.98 & 4.76 & 4.35 & 24.24 & 24.66 & 13.85 & 13.99 \\
\hline $\mathrm{S}_{9}$ & $\mathrm{C}_{22} \mathrm{H}_{22} \mathrm{~N}_{8} \mathrm{O}_{2} \mathrm{~S}_{2}$ & 53.44 & 52.96 & 4.45 & 4.51 & 22.67 & 23.08 & 12.95 & 13.04 \\
\hline \hline $\mathrm{S}_{10}$ & $\mathrm{C}_{28} \mathrm{H}_{22} \mathrm{~N}_{8} \mathrm{~S}_{2}$ & 62.92 & 60.01 & 4.11 & 4.58 & 20.97 & 21.54 & 11.98 & 10.77 \\
\hline \hline
\end{tabular}

The spectroscopic observation of $(\mathrm{S})_{2}$ is given show the disappearance of $\mathrm{NH}_{2}$ bands and the appearance of bands at 3110, 2724, 1621,1602 and 841.1 which could be assigned to $v \mathrm{C}-\mathrm{H}$ of azomethane $\operatorname{group}^{(11)}, v \mathrm{~S}-\mathrm{H}$, $v \mathrm{CH}=\mathrm{N}, v \mathrm{C}=\mathrm{C}$ and out of plane bending of para-disustituted benzene ring.

${ }^{1} \mathrm{H}-\mathrm{NMR}$ (DMSO- $\left.\mathrm{d}_{6}\right), \quad \delta$ in $\left.\mathrm{ppm}\right)$ for compouns $(\mathrm{S})_{2}$ : 7.61-7.50 (d-d, 12H, arom. H), $9.2(\mathrm{~s}, 2 \mathrm{H}, \mathrm{CH}=\mathrm{N}), 12.6(\mathrm{~s}, 2 \mathrm{H}, \mathrm{SH})$. Table (5) shows the FT-IR absorption bands for synthesized compounds.
Compounds $\left(\mathrm{D}_{1}-\mathrm{D}_{11}\right)$ were synthesized by the reaction of Schiff base compounds $\left(\mathrm{S}_{1}-\mathrm{S}_{10}\right)$ with isatin in ethanol is turn collapses to the 7-membered heterocyclic system to give substituted $[1,2,4]$ triazolo $[3,4-b][1,3]$ diazepine derivatives compounds.

The elemental analysis of compounds $\left(D_{1}-D_{11}\right)$ are listed in Table (5). 
Table (5)

Elemental analysis (CHNS-O) for compounds $\left(D_{1}-D_{11}\right)$.

\begin{tabular}{|c|c|c|c|c|c|c|c|c|c|}
\hline \multirow{2}{*}{$\begin{array}{c}\text { Comp. } \\
\text { No. }\end{array}$} & \multirow{2}{*}{ Formula } & \multicolumn{2}{|c|}{$\% \mathrm{C}$} & \multicolumn{2}{|c|}{$\% \mathrm{H}$} & \multicolumn{2}{|c|}{$\% \mathrm{~N}$} & \multicolumn{2}{|c|}{$\% \mathrm{~S}$} \\
\hline & & Calc. & Found & Calc. & Found & Calc. & Found & Calc. & Found \\
\hline $\mathrm{D}_{1}$ & $\mathrm{C}_{42} \mathrm{H}_{30} \mathrm{~N}_{10} \mathrm{O}_{4} \mathrm{~S}_{2}$ & 62.84 & 64.11 & 3.74 & 3.25 & 17.45 & 19.96 & 7.98 & 7.22 \\
\hline $\mathrm{D}_{2}$ & $\mathrm{C}_{38} \mathrm{H}_{30} \mathrm{~N}_{10} \mathrm{O}_{4} \mathrm{~S}_{2}$ & 60.47 & 58.88 & 3.97 & 4.98 & 18.56 & 17.87 & 8.48 & 7.05 \\
\hline$\overline{\overline{D_{8}}}$ & $\overline{\mathrm{C}_{40} \mathrm{H}_{24} \mathrm{~N}_{12} \mathrm{O}_{8} \mathrm{~S}_{2}}$ & 55.55 & 56.25 & 2.77 & 2.94 & 19.44 & 19.94 & 7.40 & 5.86 \\
\hline $\begin{array}{l}\mathrm{D}_{9} \\
\end{array}$ & $\begin{array}{l}\mathrm{C}_{36} \mathrm{H}_{24} \mathrm{~N}_{12} \mathrm{O}_{8} \mathrm{~S}_{2} \\
\end{array}$ & $\overline{52.94}$ & 53.32 & 2.94 & 3.19 & 20.58 & 21.23 & 7.84 & 6.68 \\
\hline $\mathrm{D}_{10}$ & $\mathrm{C}_{40} \mathrm{H}_{24} \mathrm{~N}_{10} \mathrm{O}_{4} \mathrm{~S}_{2} \mathrm{Br}_{2}$ & 51.51 & 52.37 & 2.57 & 3.10 & 15.02 & 17.65 & $\overline{6.86}$ & 8.03 \\
\hline $\mathrm{D}_{11}$ & $\mathrm{C}_{36} \mathrm{H}_{24} \mathrm{~N}_{10} \mathrm{O}_{4} \mathrm{~S}_{2} \mathrm{Br}_{2}$ & 48.87 & 50.06 & 2.71 & 3.31 & 15.84 & 16.25 & $\overline{7.24}$ & 6.95 \\
\hline
\end{tabular}

The transformation is confirmed by shows the appearance of new bands at 1724, 1693 which could be attributed to $(\mathrm{C}=\mathrm{O}$ of ketone stretching) and ( $\mathrm{C}=\mathrm{O}$ of lactam stretching) as well as the appearance of $\mathrm{N}-\mathrm{H}$ stretching band at 3280 .

6.95-8.31 (d-d, 16H, arom. H), 3.91(s, 4H, $\left.\mathrm{CH}_{2}-\mathrm{CH}_{2}\right), 10.91(\mathrm{~s}, 2 \mathrm{H}, \mathrm{SH}), 3.78(\mathrm{~s}, 6 \mathrm{H}$, $\left.\mathrm{OCH}_{3}\right)$.

\section{References}

[1] Chescman H. and Grenberg S., "Synthesis and Characterization of 5, 6-dihydro - 7H - pyrrolo [1,2d] [1,4] benzodiazepine-6-one", J. Heterocyclic Chem., 16, 241-247, 1979.

[2] Ashton H., "The diagnosis and management of benzodiazepine dependence", Psychiatry, 18, 249-255, 2005.

[3] Saoud S., "Synthesis and Characterization of Some New 1,3- Oxazepine Derivatives", IBN AL- HAITHAM J. FOR PURE \& APPL. SCI., 24 (1), 98-104, 2011.

[4] Hussein F., Obaid H., Sabah. T., "Synthesis and characterization of 2- aryl3-(p-methoxy phenyl)-2,3-dihydro-1,3oxazepine-4,7-diones", Irq. J. Chem., 27, 751-763, 2001.

[5] Hussein F., Sabah. T., "Synthesis and characterization of 2, 3-diaryl- 2,3dihydrobenz [1,2-e] [1,3] oxazepine-4,7diones", Irq. J. Chem., 28, 13-25, 2002.

[6] Ali K., Ph. D. Thesis, University of Baghdad, 2005.

[7] Huseein D., MSc. Thesis, University of Baghdad, 2006.

[8] Abdul-Razzak F., Ph. D. Thesis. University of Baghdad, 2006.

[9] Jubie S., Sikder p., Antony S., Kalira Jan R., Gowramma B., Gomathy S. and Elango K., "Synthesis and biological evaluation of some schiff bases of [4-(Amino) -5Phenyl- 4H-1, 2, 4-Triazole- 3- Thiol] [Synthesis and biological evaluation of some schiff bases of [4-(Amino) -5Phenyl- 4H-1, 2, 4-Triazole- 3- Thiol]", Pak.J. Pharm. Sci., 24, 109-112, 2011.

[10] Olcay B. and Hakan B.. Molecules, 13, 2126-2135 (2008).

[11] Silverstein R., Francis X., David J., David L., "Spectrometric Identification of Organic Compounds", Wiley, 8th Edition, 2015.

[12] Al-fatahi Y., Nasreen J., Al-Razak A., "Synthesis of new bis[1,2,4]triazolo[3,4$b][1,3,4]$ thiadiazoles", Journal of College of Education, Al-Mustansiriya University, 1, 38-45, 2008. 